\title{
UCST and LCST Behavior in Polymer Blends and Its Thermodynamic Interpretation
}

\author{
Toshiaki OUGIZAWA and Takashi INOUE* \\ Department of Textile and Polymeric Materials, Tokyo Institute of Technology \\ Ookayama, Meguro-ku, Tokyo 152, Japan
}

(Received December 26, 1985)

\begin{abstract}
A pair of dissimilar polymers with high molecular weights, poly(acrylonitrile-costyrene)/poly(acrylonitrile-co-butadiene), was found to exhibit both UCST (upper critical solution temperature) and LCST (lower critical solution temperature) behavior. This phase behavior is interpreted by combining the solubility parameter theory involving the free volume term and the recent idea of "miscibility window" for the polymer blends including random copolymers.

KEY WORDS Upper Critical Solution Temperature / Lower Critical

Solution Temperature / Random Copolymer / Blends / Equation-of-state /

Miscibility Window / $\chi$-Parameter / Solubility Parameter /
\end{abstract}

It is well known that most pairs of dissimilar polymers with high molecular weights are immiscible. This is so because the combinatorial entropy of mixing of two polymers is dramatically smaller than that of two low molecular weight compounds and the enthalpy of mixing is often a positive quantity. ${ }^{1}$ Therefore dissimilar polymers are only miscible if there are favorable specific interactions between them leading to a negative contribution to the Gibbs free energy of mixing. Miscible polymer-polymer mixtures tend to phase-separate at elevated temperatures. This LCST (lower critical solution temperatures) behavior is typical for miscible polymer blends. The LCST behavior is interpreted in terms of equation-of-state or free volume contribution. ${ }^{2,3}$ Some miscible polymer blends also exhibit phase separation at low temperatures. This UCST (upper critical solution temperature) behavior is rather uncommon. It has been observed only when one or two components have relatively low molecular weight, i.e., oligomer. ${ }^{4,5}$ So far, the

\footnotetext{
* To whom correspondence should be addressed.
}

blends of dissimilar polymers with high molecular weights have been found to show only the LCST behavior.

We recently found that a pair of dissimilar polymers with high molecular weights, polybutadiene/poly(styrene-co-butadiene), exhibited both UCST and LCST behavior. ${ }^{6}$ To our knowledge, this is the first observation of the coexistence of UCST and LCST behavior in polymer blends of high molecular weight polymers. ${ }^{24}$. In this paper, we describe the results with another system exhibiting both UCST and LCST behavior, poly(acrylonitrileco-styrene)/poly(acrylonitrile-co-butadiene).

We also present. the thermodynamic interpretation of this phase behavior by combining the equation-of-state theory ${ }^{7-9}$ and the recent idea of "miscibility window" for the polymer blends including random copolymers. ${ }^{10-12}$

\section{EXPERIMENTAL}

Polymer specimens used in this study, AS25 and NBR-40, were commercial polymers. 
The AS-25 is poly(acrylonitrile-co-styrene) containing $25 \mathrm{wt} \%$ acrylonitrile, supplied by the Mitsubishi Monsant Co., Ltd. (SAN-C, $\left.M_{n}=6.84 \times 10^{4}, M_{w}=1.94 \times 10^{5}\right)$. The NBR-40 is poly(acrylonitrile-co-butadiene) containing $40 \mathrm{wt} \%$ acrylonitrile, supplied by the Japan Synthetic Rubber Co., Ltd. (N220S, $M_{n}=$ $\left.9.13 \times 10^{4}, \quad M_{w}=2.97 \times 10^{5}\right)$. These molecular weights were estimated by gel permeation chromatography calibrated with polystyrene standard. Note here that both polymers are random copolymers.

The AS-25 and NBR-40 were dissolved at $8 \mathrm{wt} \%$ of total polymer in tetrahydrofuran. The solution was cast onto a cover glass (for microscopy). The rate of solvent evaporation was adjusted to prepare the cast film with a regularly phase separated structure. ${ }^{13}$ The cast film was further dried under vacuum of $10^{-4} \mathrm{mmHg}$ for $10 \mathrm{~h}$.

The structure of the dried film was observed under a light microscope. The film on the cover glass was inserted in a heating stage, the Linkam TH600 Heating-cooling stage. ${ }^{14}$ This stage can be programmed to provide isothermal setting and also a linear rise in temperature at any of 27 different rates between $0.1 \mathrm{~K} \mathrm{~min}^{-1}$ to $90 \mathrm{~K} \mathrm{~min}^{-1}$. The heating stage was set horizontally to the light scattering stage. ${ }^{13}$ A goniometer trace of the scattered light from the film was given during heating at a constant rate. Similar experiments were carried out at different heating rates and isothermal settings.

\section{RESULTS AND DISCUSSION}

Figure 1 is a typical example of the light micrograph of the cast film of AS-25/NBR$40=50 / 50$. A highly interconnected two-phase morphology with uniform domain size is seen in the micrograph. We call it a "modulated structure,"13 partly for convenience to describe the morphological features of unique periodicity and high level of phase connectivity. Figure 2 shows a light scattering pat-

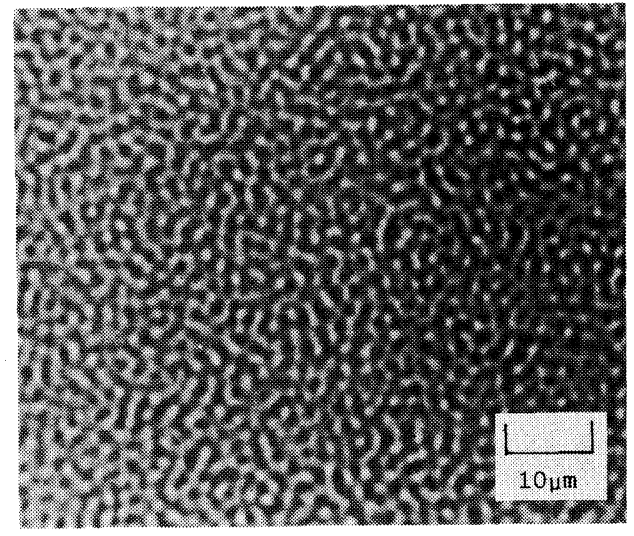

Figure 1. Light micrograph of AS-25/NBR-40 $=50 / 50$.

tern (a) and a light scattering profile (b) from the cast film of Figure 1. The ring pattern indicates some degree of regularity of the phase separated structure in Figure 1. The Bragg spacing from the peak in the light scattering profile corresponds to the periodic distance in Figure 1.

Elevating the temperature at a constant rate, the light scattering profile did not change up to a certain temperature $T_{\mathrm{d}}$. Above $T_{\mathrm{d}}$ the intensity of the scattering peak decreased with temperature, keeping peak angle almost constant. The scattering peak finally disappeared. $T_{\mathrm{d}}$ corresponds to onset temperatures of phase dissolution, as will be discussed later. $T_{\mathrm{d}}$ varied with heating rate. Figure 3 shows $T_{\mathrm{d}}$ $v s$. heating rate plots. Intercept of $T_{\mathrm{d}}$, at which the heating rate is zero, may correspond to the binodal temperature. Similar experiments were carried out for blend specimens with various compositions. The binodal points thus estimated are indicated by open circles in Figure 4. A UCST-type phase diagram is drawn in Figure 4. However, it is quite strange in shape. In the AS-25 rich region, $T_{\mathrm{d}}$ (intercept) linearly decreases with composition. This behavior should be apparent because of the location of glass transition temperature $\left(T_{\mathrm{g}}\right)$.

A $T_{\mathrm{g}} v s$. composition curve estimated by the simple weight-average is shown by a thin solid 


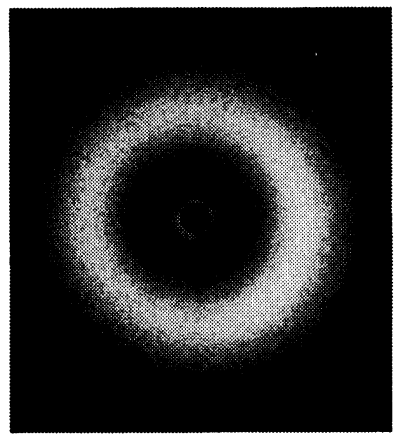

(a)

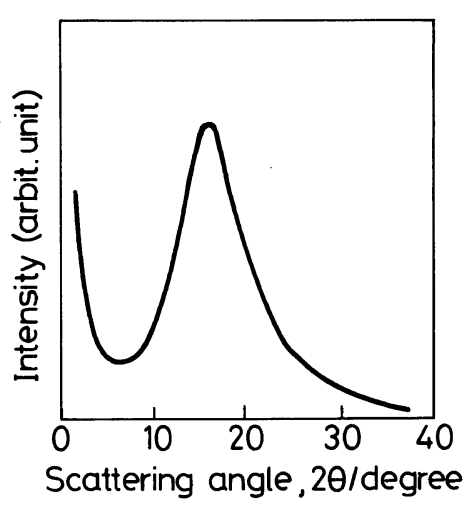

(b)

Figure 2. (a) Light scattering pattern ( $\left.V_{\mathrm{v}}\right)$ from AS- $25 / \mathrm{NBR}-40=50 / 50$ blend film of Figure 1 , and (b) its goniometer trace. A He-Ne laser of $632.8 \mathrm{~nm}$ wavelength was radiated.

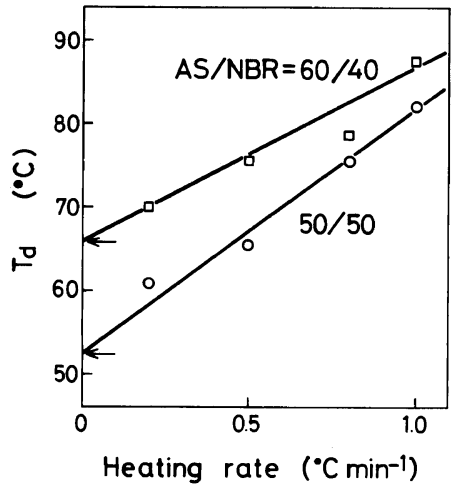

Figure 3. $T_{\mathrm{d}} v s$. heating rate plots. $T_{\mathrm{d}}$ is the onset temperature of the scattered intensity decay.

line in Figure 4. The observed $T_{\mathrm{d}}$ (intercept) in the AS-25 rich region is just above the $T_{\mathrm{g}}$ line and the former is parallel to the latter. Below $T_{\mathrm{g}}$, of course, the displacement of chain molecules is almost immobilized. Hence, the phase dissolution cannot take place below $T_{\mathrm{g}}$ even when the system is thrust into the single phase region above UCST. Even above $T_{\mathrm{g}}$, at temperatures just above $T_{\mathrm{g}}$, the rate of phase dissolution may be so low that one cannot detect this phenomenon in a few hours. Real binodal may be such as represented by a dotted line in Figure 4.

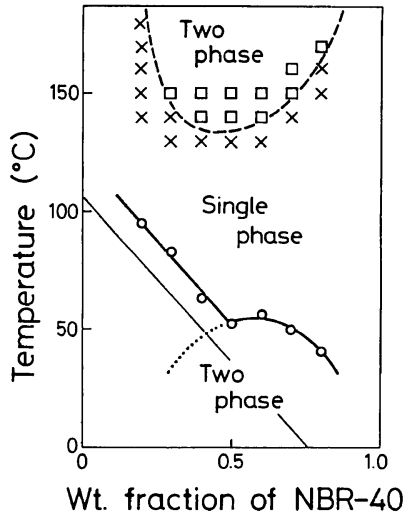

Figure 4. Phàse diagram of AS-25/NBR-40 system. Open circles are the $T_{\mathrm{d}}$ at zero heating rate. Thin solid line is the $T_{\mathrm{g}}$ curve calculated by the weight-average; $T_{\mathrm{g}}=107^{\circ} \mathrm{C}$ for AS-25, $T_{\mathrm{g}}=-33^{\circ} \mathrm{C}$ for NBR-40. UCST curve is continued symmetrically into the AS-25 rich region as indicated by dotted line. LCST curve is shown by broken line; $(\square)$ the film became opaque and $(x)$ was still clear after the temperature-jump from single phase region.

The UCST behavior was confirmed by isothermal experiments as follows. Film specimens with modulated structures underwent a rapid temperature-jump from room temperature to various higher temperatures set isothermally and above the UCST. Below UCST, appreciable change in the scattering profile with 


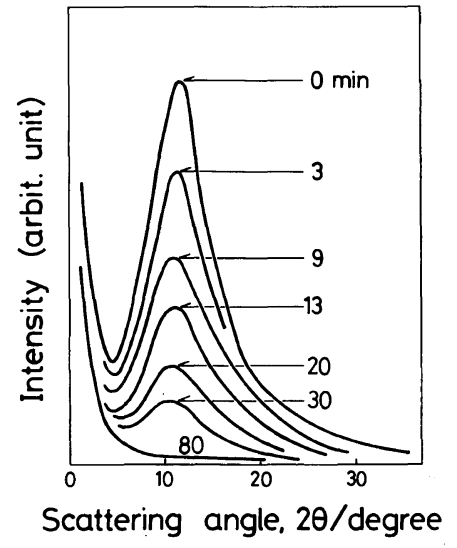

Figure 5. Change of light scattering profile with annealing at $85^{\circ} \mathrm{C}$. AS-25/NBR-40 $=50 / 50$. Each numeral gives the time after temperature-jump.

time of isothermal annealing could not be detected in $2 \mathrm{~h}$. This means that appreciable structural change did not take place. On the other hand, above UCST, the scattered intensity decreased with time of annealing, keeping the peak angle constant. A typical example is shown in Figure 5. The intensity decay in Figure 5 corresponds to the phase dissolution of the modulated structure, keeping the periodic distance constant. ${ }^{6}$ This was also confirmed under a light microscope. Below UCST, the modulated structure did not change with time after the temperature-jump. Above UCST, the image contrast of modulated structure became weak gradually, and finally the image disappeared. The kinetic aspect of the phase dissolution is an interesting problem. It is however out of the scope of this paper and presented elsewhere. ${ }^{15}$

For the investigation of phase behavior at higher temperatures, the gradual heating procedure used for the study of UCST was not appropriate. It was due to deterioration of specimens by long exposure to high temperature atmosphere during the slow heating process. Instead we employed a dissolution and temperature-jump procedure: the cast film was annealed above UCST, e.g., at $90^{\circ} \mathrm{C}$ for ca. $60 \mathrm{~min}$, then the annealed specimen underwent a rapid temperature-jump to an isothermal setting at higher temperature and the structural change was observed under a microscope and by the light scattering technique. When no appreciable change took place after the temperature-jump, we judged that the system was still at the single phase region. On the other hand, when the film became opaque and the scattering intensity increased, we judged that the system was at the two phase region. On the basis of these observations, the LCST line was drawn somewhat arbitrarily by a broken line in Figure 4 . Actually, we observed the development of a modulated structure during isothermal annealing at the two phase region above LCST. It was similar in appearance to that in Figure 1 but the periodic distance of the structure was much shorter than the original one in the ascast film. This implies that the structural memory in the cast film had disappeared by annealing at the single phase region and a new concentration fluctuation developed by the spinodal decomposition thermally induced above LCST.

Thus we have found the coexistence of UCST and LCST behavior in AS-25/NBR-40 system.

\section{THERMODYNAMIC DISCUSSION}

The coexistence of UCST and LCST is not specific to this particular pair, AS-25/NBR-40. Similar phase behavior has been also found in another pair of high molecular weight polymers, polybutadiene/poly(styrene-co-butadiene), ${ }^{6}$ and seems to be rather general phenomenon for some classes of homopolymer/ copolymer and copolymer/copolymer systems. However, this phase behavior has never been predicted by current theories of polymerpolymer miscibility. ${ }^{2,3,16-18}$

The equation-of-state theory successfully interprets the LCST behavior for the mixture with specific interactions leading to a negative 
heat of mixing (exothermic system). For the mixture with repulsive interactions leading to a positive heat of mixing (endothermic system), this theory interprets the appearance of UCST and LCST, if the molecular weights of component polymers are so small that the combinatorial entropy of mixing makes a significant contribution to the Gibbs free energy of mixing. However, when the molecular weights are very high, the appearance of UCST, both in the endothermic and exothermic systems, is not interpreted directly by the current equation-of-state theory.

Here we will describe a thermodynamic interpretation of the coexistence of UCST and LCST behavior by combining the recent idea of "miscibility window" and the solubility parameter theory involving the free volume term in the equation-of-state theory.

Neglecting the combinatorial entropy term for the mixture of dissimilar polymers with high molecular weights, the miscibility problem is simply an argument on the sign of the Flory's interaction parameter $\chi_{12}$; if $\chi_{12}$ is negative, the dissimilar polymers are miscible. ${ }^{1}$

For a binary system of the copolymer-1 composed of monomers $\mathrm{A}$ and $\mathrm{B}$ and the copolymer- 2 composed of monomers $C$ and $D$, the interaction parameter is given by

$$
\begin{aligned}
\chi_{12}= & x y \chi_{\mathrm{AC}}+(1-x) y \chi_{\mathrm{BC}} \\
& +x(1-y) \chi_{\mathrm{AD}} \mid+(1-x)(1-y) \chi_{\mathrm{BD}} \\
& -x(1-x) \chi_{\mathrm{AB}}-y(1-y) \chi_{\mathrm{CD}}
\end{aligned}
$$

where $x$ and $y$ are the copolymer compositions in volume fraction of $\mathrm{A}$ and $\mathrm{C}$, respectively, i.e., copolymer-1 is $\left(\mathrm{A}_{x} \mathrm{~B}_{1-x}\right)$ and copolymer2 is $\left(C_{y} D_{1-y}\right)$. Even if all binary interactions, $\chi_{\mathrm{AC}}, \chi_{\mathrm{BC}}, \chi_{\mathrm{AD}}, \chi_{\mathrm{BD}}, \chi_{\mathrm{AB}}$, and $\chi_{\mathrm{CD}}$, are positive, the net interaction $\chi_{12}$ can be negative in a limited range of $x$ and $y$. This happens when $\chi_{\mathrm{AB}}$ and/or $\chi_{\mathrm{CD}}$ are very large. Thus, the apparent attractive interaction between copolymers 1 and 2 comes from the large repulsive interaction between comonomers $\mathrm{A}$ and $\mathrm{B}$ (and/or C and D). This is the concept of
Table I. Values of $\alpha$ and $\delta$ from the polymer handbook

\begin{tabular}{lccc}
\hline \multirow{2}{*}{ Polymer } & \multicolumn{1}{c}{$\alpha$} & $\delta\left(25^{\circ} \mathrm{C}\right)$ \\
\cline { 2 - 3 } & ${ }^{\circ} \mathrm{C}^{-1}$ & & $\left(\mathrm{cal} / \mathrm{cm}^{-3}\right)^{1 / 2}$ \\
\hline (A) Polyacrylonitrile & $1.4 \times 10^{-4}$ & 12.75 \\
(B) Polystyrene & $1.9 \times 10^{-4}$ & 9.12 \\
(D) Polybutadiene & $7.1 \times 10^{-4}$ & 8.38 \\
\hline
\end{tabular}

"miscibility window,"7-9 which interprets miscibility without any specific interactions.

When there are no specific interactions such as hydrogen bonding and charge transfer complex, $\chi_{i j}$ may be represented by

$$
\chi_{i j}=\frac{V_{i}}{R T}\left(\delta_{i}-\delta_{j}\right)^{2}
$$

where $V_{i}$ is the molar volume of $i$-component, $T$ is temperature, $R$ is gas constant, and $\delta$ is the solubility parameter defined by $\delta=$ $\left(\Delta E^{V} / V\right)^{1 / 2}$, where $\Delta E^{V}$ is the energy of vaporization. ${ }^{19}$

The familiar formulation by eq 2 is commonly assumed to involve only energetic interaction. This is so when there is no volume change with mixing $\left(\Delta V_{\mathrm{m}}=0\right)$. However, if we eliminate the condition of $\Delta V_{\mathrm{m}}=0$, the equation-of-state or the free volume term is automatically involved in eq 2 . Namely, $\delta$ depends on the free volume through $\Delta E^{V}$ and $V$. This point has been suggested by Biros et al. $^{20}$ in terms of

$$
\left(\frac{\partial \ln \delta}{\partial T}\right)_{P} \simeq-\alpha
$$

where $\alpha$ is thermal expansion coefficient. Hence, $\chi_{i j}$ by eq 2 implicitly involves the free volume contribution.

From eq 1 and $2, \chi_{12}$ is given by

$$
\begin{aligned}
\chi_{12}= & \frac{V_{1}}{R T}\left\{x y\left(\delta_{\mathrm{A}}-\delta_{\mathrm{C}}\right)^{2}+(1-x) y\left(\delta_{\mathrm{B}}-\delta_{\mathrm{C}}\right)^{2}\right. \\
& +x(1-y)\left(\delta_{\mathrm{A}}-\delta_{\mathrm{D}}\right)^{2}+(1-x)(1-y)\left(\delta_{\mathrm{B}}-\delta_{\mathrm{D}}\right)^{2} \\
& \left.-x(1-x)\left(\delta_{\mathrm{A}}-\delta_{\mathrm{B}}\right)^{2}-y(1-y)\left(\delta_{\mathrm{C}}-\delta_{\mathrm{D}}\right)^{2}\right\}^{\prime}
\end{aligned}
$$




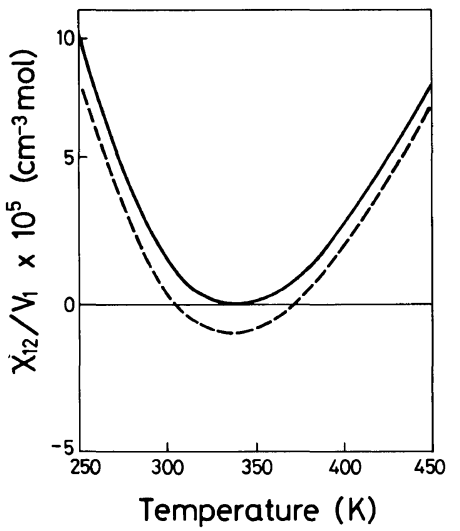

Figure 6. Temperature dependences of $\chi_{12}$ calculated; by eq 4 (solid line); by eqs 4 and 6 (broken line) setting $\kappa_{\mathrm{BD}}=-1 \times 10^{-4}$ and $\kappa_{\mathrm{AD}}=\kappa_{\mathrm{AB}}=0$.

Combining eq 3 and 4 , one can estimate the temperature dependence of $\chi_{12}$, provided that the values of $\alpha_{i}$ and $\delta_{i}$ (at $25^{\circ} \mathrm{C}$ ) are given. For our AS-25/NBR-40 system, we are able to pick up these values from Polymer Handbook. ${ }^{21}$ They are summarized in Table I.

The temperature dependence of $\chi_{12}$ calculated for AS-25/NBR-40 is shown by the solid line in Figure 6. The temperature dependence of $\chi_{12}$ is concave. Minimum $\chi_{12}$ is zero but not negative, as is natural. The situation is revealed by rewriting eq 4 by

$$
\chi_{12}=\frac{V_{1}}{R T}\left(\delta_{1}-\delta_{2}\right)^{2}
$$

where

$$
\delta_{1}=x \delta_{\mathrm{A}}+(1-x) \delta_{\mathrm{B}}
$$

and

$$
\delta_{2}=y \delta_{\mathrm{C}}+(1-y) \delta_{\mathrm{D}}
$$

and by plotting the temperature dependence of $\delta_{1}$ and $\delta_{2}$ as in Figure 7.

Further, if one recalls that eq 2 comes from the assumption of the so-called "geometric mean rule" for the contact energies $\varepsilon_{i i}$ and $\varepsilon_{j j}$ $\left(\varepsilon_{i j} \cong \sqrt{\varepsilon_{i i} \cdot \varepsilon_{j j}}\right),{ }^{22,27}$ eq 2 may be formulated more generally as

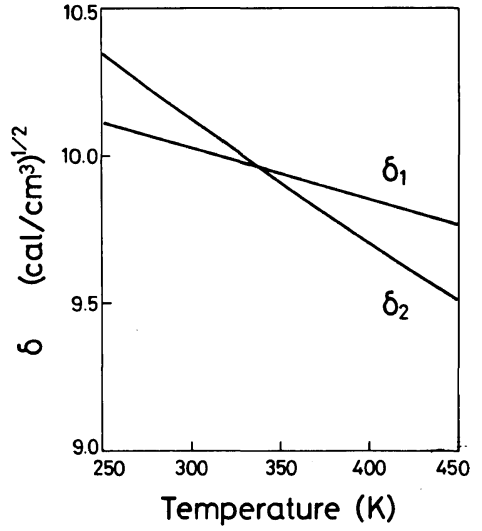

Figure 7. Temperature dependences of $\delta$ calculated. $\delta_{1}$ and $\delta_{2}$ are the solubility parameters of AS-25 and NBR40 , respectively.

$$
\chi_{i j}=\frac{V_{i}}{R T}\left\{\left(\delta_{i}-\delta_{j}\right)^{2}+2 \kappa_{i j} \delta_{i} \delta_{j}\right\}
$$

where $\kappa_{i j}$ is a parameter describing the departure from the geometric mean rule. ${ }^{12}$ Combining eq 4 and 6 and introducing a very slight deviation $\left(\right.$ e.g., $\kappa_{\mathrm{BD}}=-1 \times 10^{-4}$, $\kappa_{\mathrm{AD}}=\kappa_{\mathrm{AB}}=0$, for the A-B copolymer/A-D copolymer system $\left.{ }^{23}\right), \chi_{12}$ shifts downward to negative values at a certain temperature range, as shown by the broken line in Figure 6.

Thus, we are able to interpret the appearance of both UCST and LCST. The interpretation is just a semi-empirical one. However, the result is quite interesting. Of particular interest is answer to the question: what is the physical meaning of the concave character in the temperature dependence of $\chi_{12}$ ? An answer may be as follows.

As has been usually done, let's separate $\chi_{12}$ into the interactional energy term $\left(\chi_{\text {int }}\right)$ and the free volume term $\left(\chi_{\text {free }}\right)$. The equation-of-state theory has shown that $\chi_{\text {free }}$ is an increasing function of temperature as shown in Figure 8a. So the total $\chi_{12}$ could be concave with minimum, if $\chi_{\text {int }}$ changes sign from positive to negative with increasing temperature as shown in Figure 8a. However, such temperature dependence of $\chi_{\text {int }}$ is not conceivable. 


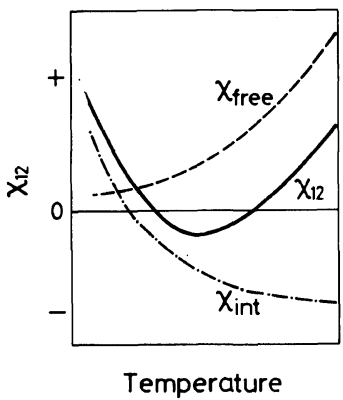

(a)

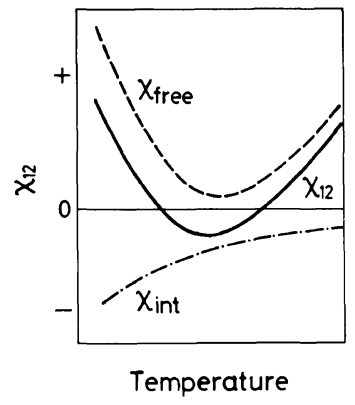

(b)
Figure 8. Schematic representation for the constitution of $\chi_{12}(T)$ : (a) familiar $\chi_{\mathrm{free}}(T)$ and unconceivable $\chi_{\text {int }}(T)$, (b) conceivable $\chi_{\text {int }}(T)$ and $\chi_{\text {free }}(T)$ to be investigated theoretically in the future.

The natural temperature dependence of $\chi_{\text {int }}$ should be given as shown in Figure $8 \mathrm{~b}$. If it is so, $\chi_{\text {free }}$ itself should be concave. In other words, the free volume contribution becomes bigger also with decreasing temperature. This behavior has never been predicted by the current theories. However, we believe that it may be so for polymer-polymer mixtures, but not so for polymer-solvent systems.

Acknowledgements. We acknowledge the partial support by a Grant-in-Aid for Scientific Research (No. 60470103) from the Ministry of Education, Science, and Culture of Japan, and a grant from the Japan Synthetic Rubber Co., Ltd., Tokyo, Japan.

\section{REFERENCES AND NOTES}

1. P. J. Flory, "Principles of Polymer Chemistry," Cornell University Press, Ithaca, New York, 1953.

2. D. R. Paul and S. Newman, Ed., "Polymer Blends," Academic Press, New York, N. Y., 1978.

3. O. Olabishi, L. M. Robeson, and M. T. Shaw, "Polymer-Polymer Miscibility," Academic Press, New York, N. Y., 1979.
4. R. J. Roe and W. C. Zin, Macromolecules, 13, 1221 (1980).

5. S. L. Zacharius, G. ten Brinke, W. J. NacKnight, and F. E. Karasz, Macromolecules, 16, 381 (1983).

6. T. Ougizawa, T. Inoue, and H. W. Kammer, Macromolecules, 18, 2089 (1985).

7. P. J. Flory, R. A. Orwoll, and A. Vrij, J. Am. Chem. Soc., 86, 3507, 3515 (1964).

8. P. J. Flory, J. Chem. Phys., 87, 1833 (1965).

9. D. Patterson and A. Roberd, Macromolecules, 11, 690 (1978).

10. R. P. Kambour, J. T. Bendler, and P. C. Bopp, Macromolecules, 16, 753 (1983).

11. G. ten Brinke, F. E. Karasz, and W. J. MacKnight, Macromolecules, 16, 1827 (1983).

12. D. R. Paul and J. W. Barlow, Polymer, 25, 487 (1984).

13. T. Inoue, T. Ougizawa, O. Yasuda, and $\mathrm{K}$. Miyasaka, Macromolecules, 18, 57 (1985).

14. T. Shephered, Econ. Geol., 76, 1244 (1981).

15. T. Ougizawa, Y. Takagi, and T. Inoue, Polym. Prepr., Jpn., 34, 771 (1985); submitted to Polymer.

16. I. C. Sanchez and R. H. Lacombe, J. Phys. Chem., 80, 2352 (1976).

17. R. H. Lacombe and I. C. Sanchez, J. Phys. Chem., 80, 2568 (1976).

18. I. C. Sanchez and R. H. Lacombe, Macromolecules, 11, 1145 (1978).

19. J. H. Hildebrand and R. L. Scott, "The Solubility of Non-Electrolytes," 3rd ed, Reinhold, New York, N.Y., 1950.

20. J. Biros, L. Zeman, and D. Patterson, Macromolecules, 4, 30 (1971).

21. J. Brandrup and E. H. Immergut, Ed., "Polymer Handbook," Interscience, New York, N. Y., 1967.

22. F. London, Z. Physik, 63, 245 (1930).

23. $K_{i j}$ are measurable quantities. Experiments are under way.

24. Referee let us know that UCST behavior has been observed in polystyrene/poly(vinyl methyl ether) system. ${ }^{25,26}$

25. T. K. Kwei, T. Nishi, and R. F. Roberts, Macromolecules, 7, 667 (1974).

26. J. M. G. Cowie and S. Saeki, Polym. Bull., 6, 75 (1981).

27. $\varepsilon_{i j}$ is the energy of $i-j$ contact. $\varepsilon_{i i}$ is related to $\left(\Delta E^{v}\right)_{i}$ by $\varepsilon_{i i}=\left[\left(\Delta E^{v}\right)_{\mathrm{i}} / V_{\mathrm{i}}\right](2 / A Z)$, where $A$ is Avogadro's number, $Z$ is the coordination number, and $V_{i}$ is the molar volume of $i$-component. 